\title{
Analysis of High Impedance Surface as Ground of Monopole Antenna for Bandwidth Enhancement
}

\author{
Fatimah Zaharah Ali \\ Faculty of Electrical Engineering \\ University of Technology MARA (UiTM) \\ Permatang Pauh, Penang, Malaysia \\ fatimah_zaharah@ppinang.uitm.edu.my
}

\begin{abstract}
Monopole antenna is one of the famous low-profile antennas because of its features of light in weight and easy to install. However, it can only be operated in lower gain and bandwidth due to the easiness of noise affecting. As other types of antenna, monopole antenna uses perfectly electrical conducting plane (PEC) as its ground plane which are made by a printed circuit board (PCB). PCB is well-known with its disadvantages in terms of bandwidth, loss, and gain. Thus, in order to enhance the performance of a monopole antenna, it is good if the radiation's part is undisturbed in order to maintain the antenna's features, while only at the ground is focused. In this project, a structure with arrays of protrusions instead of flat plate that is commonly used on most ground plane will be developed. This structure is called as High Impedance Surface (HIS) ground plane. It has been proved that a corrugated metal surface (protrusions) where the corrugations are folded up into lumped-circuit elements can have high impedance value on the surface. HIS is believed to eliminate the surface waves which reduce the power radiation as well as the efficiency. Therefore, the bandwidth frequency can be enhanced and antenna's performance can be improved. In this report, some specifications such as length of antenna, width, and others required parameters are shown. These parameters are used to remodel the ground plane of the antenna according to the desired one where it can operate in lower frequency but still can perform well in term of higher bandwidth and efficiency of transmission.
\end{abstract}

Keywords-high impedance surface; electromagnetic bandgap; perfectly electrical conducting plane; printed circuit board; monopole antenna

\section{INTRODUCTION}

Antennas may be 'stand-alone', but some of them need a ground to be mounted with for protection from lightning. Depending on the applications, the antenna can be connected to a coaxial connector or conductive plane for grounding. The conductive plane or ground plane is made from printed circuit board (PCB) that has flat copper layer on both side of the plate. Commonly, this plate is called as perfectly electric conducting (PEC) plate due to its characteristics of conductive.

\section{A. Ground Plane}

In some applications such as mobile phone and car that require a low profile antenna for radio frequency (RF) transmission, PEC is used as antenna's reflector. The reflector is important for some antenna's design because it is able to block the other half of power source which means the radiation signal that comes towards it will be reflected back to the antenna. This leads to the increasing of gain and power on the antenna and more signals will flow through it. A ground plane is usually a flat copper plate which has an ability to carry currents due to its nature of conductive. The currents that flow on ground plane are typically reverse in direction and they are named as image currents.

These image currents bring disadvantage to the antenna because when the ground plane is placed close enough to the radiator, the real currents that flow on the antenna tend to be cancelled out by the image current on the ground plane. This is because the image currents formed on the ground plane are typically similar amount with the real currents. Besides, the image currents are in opposite direction of the real currents. Note that when the real currents on the antenna are cancelled out, the power source will be dissipated. As the result, the radiation efficiency becomes poor as the gain and bandwidth will be reduced. Thus, D. Sievenpiper in his report (1999) [5] has stated that the antenna and the ground plane must be distanced at least quarter of the wavelength, i.e; $\lambda / 4$, away.

The image currents brought by the ground plane are normally the surface currents. D. Sievenpiper with collaboration with other researchers (1999) [5] have mentioned in their report that the surface currents will be described as surface waves at radio frequency (RF). Therefore, the ground plane suffers with the surface waves. These surface waves are the most unwanted waves in antenna's design because it could weaken the performance and efficiency of the radiation.

As PEC can be simply made from PCB, there is insulative composition in the middle of two conductive layers named substrate that is normally used to define the type of the PCB. The characteristic of the substrate depends on its value of dielectric constant, $\varepsilon_{\mathrm{r}}$. The lowest and the best value of $\varepsilon_{\mathrm{r}}$ is 1 which can only be found from air and anything similar. The highest the $\varepsilon_{\mathrm{r}}$ means the slower the reflected radiation signal from ground plane to move back to radiator side which is the antenna. When the reflected radiation propagates slowly to the antenna, the total of the signals to be radiated by the antenna won't be increased as expected from the usage of ground plane. Here it can be understood that the resonant frequency as well as the bandwidth will be decreased. This has been proved by the 
project conducted by Fan Yang and Yahya Rahmat Samii (2009) [15].

The thickness of the ground plane's substrate as well influences the performance of the antenna. As reported by K. Y. Sze through his analysis (1998) [16], the thicker the substrate height leads to the enhancement of frequency bandwidth around the resonant frequency.

\section{B. Low-Profile Antenna}

Low-profile antenna is well-known as a narrowband antenna which means its frequency bandwidth is lower than a normal antenna. It can be operated in lower gain and lower power. Most of the low-profile antennas such as microstrip antenna and wire antenna are desirable for most of the applications presently due to its features of simplicity, small in size, inexpensive and light. However, it limits some of the requirements such as size, radiation pattern, and bandwidth. In order to get better and efficient radiation, the size of the low-profile antenna must be made bigger and high power must be consumed. These have hinged the intention of using the small antenna.

To facilitate the external features of a low-profile antenna, the ground plane that is mounted with the antenna will be the part to be modified. There are many types of practices that can be used to enhance the performance of an antenna through ground plane such as using lower $\varepsilon_{\mathrm{r}}$, increase substrate's thickness, or apply new geometry structure of ground plane. By the new geometry structure of a ground plane, a breakthrough of a textured structure was found. It was created for photonic crystal purposes by Thomas Krauss (1996) [17] using two-dimensional (2-D) planar surfaces. The idea of the design however, was adapted from Eli Yablonovitch (1987) [18] through his invention of threedimensional (3-D) periodic optical structure. The structure is made by drilling a hole on each protrusion that has been design on a layer. Thus, D. Sievenpiper [5] has come out with a simplest and popular mushroom-like surface for textured ground plane intended for microwave transmission. The mushroom-like surface of the ground plane is a 2-D planar type [15] and the name of the new structure invented by D. Sievenpiper was electromagnetic band gap (EBG).

\section{Monopole Antenna}

Monopole antenna is one of the wire type antennas. It can simply be made by a rod of copper wire. Thus this kind of antenna is said to be under the category of low-profile antenna. Due to its simple, light and easy to install, monopole antenna has been widely used in many applications.

By using a ground plane that is wide enough, a monopole antenna could behave like a dipole antenna if it is mounted vertically on the ground plane. This is because the ground plane becomes the other missing half of the dipole antenna by reflecting back the currents flew on monopole antenna.

Typically monopole antenna's length is measured using quarter of the wavelength since most of the application used the quarter wave antenna. However, monopole antenna as well can be calculated using the custom equation of dipole antenna that has been divided by two as shown in (1).

$$
L_{\text {monopole }}=\frac{71.325}{f_{\mathrm{MHz}}}
$$

\section{Surface Waves}

As mentioned earlier, ordinary ground plane suffers with surface waves that degrade the performance of the antenna. Surface waves where it can be obtained from the leaky waves in the smooth ground plane [19] can lead to poor performance and lower efficiency of an antenna. It is because surface waves are the reverse radiation of the current that travel along the interface between the free-space and top copper layer of ground plane. These kind of unwanted waves are excited at the edges and corners of the ground plane. When the wanted waves are added with surface waves, multipath interference and back lobes radiation will be formed [23,24]. According to [5], surface waves can always occur if the ground plane used is smooth and flat. It is because such characteristics that can be obtained from PEC plate, allow surface currents to flow to infinite distance. This will also lead to the multipath interference.

\section{E. High Impedance Surface (HIS)}

To overcome the effects of surface waves, a remodeled ground plane is implemented replacing the ordinary ground plane which has characteristics of flat, smooth and continuous conductivity layer. The remodeled ground plane is characterized by having high surface impedance compared to the ordinary ground plane. With no continuous copper layer, the remodeled ground plane is developed by texturing the top copper layer by etching out some of the copper in order to form arrays of patches or protrusions. The patches will be connected to the bottom layer through conductive via and the via is fabricated by drilling and soldering the center of the protrusions to the bottom layer. This is portrayed in Figure 1 and such remodeled ground plane is called as high impedance surface (HIS) named by D. Sievenpiper [5]. However, there are a lot of applications emerged from the development of HIS. HIS was known as electromagnetic band gap (EBG) plate. It has been named as HIS because of the textured structure on top of the PCB that consists of insulative gaps that serve as surface waves 'eliminator'.

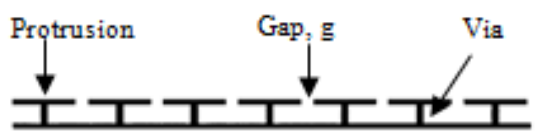

Figure 1. Cross section view of HIS.

The structure of HIS can also be folded-up into equivalent LC circuit that consists of inductance and capacitance as shows in Figure 2. From this LC circuit where the inductance and capacitance are in parallel, the total impedance can be mathematically calculated in order to prove that the result will be infinity, simultaneously proving that HIS has high surface impedance. 


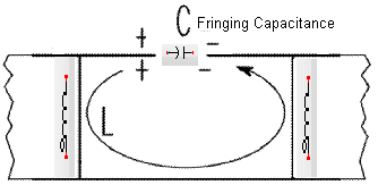

Figure 2. Equivalent LC circuit.

The gaps on the HIS are insulative in nature where they disallow the currents to pass through them. Thus the currents on the HIS ground plane cannot flow from one patch to another continually on the same layer. However, the currents have to propagate from a patch to bottom layer trough via and up to the other patches. Because of the existence of the gaps, forbidden frequency band occurs. This frequency band acts as a filter that eliminates the propagating surface waves. D. Sievenpiper et al. [5] in their project as well associated the HIS with this frequency due to the characteristic of high impedance. They also mentioned that when the forbidden frequency is close to resonant frequency, the HIS will have higher surface impedance compared to PEC. Within the band, the efficiency of the radiation will be stunning as it won't be affected by multipath interference. As the gap is associated with the capacitance as shown in Figure 2, it can be understood that the operating frequency will be higher if the gap is wider because the capacitance will definitely be lower. This has been proven by the project conducted by [5] and equation (2).

$$
f=\frac{1}{2 \pi \sqrt{L C}}
$$

\section{OBJECTIVES}

The main objective of this project is to analyze the HIS's contributions towards the low-profile antenna's performances, where in this project, the monopole antenna is used. Thus, to perform that, the sizes of the HIS's patches and plate are randomly defined. This is because there is no exact formula or equation available for HIS design. In that case, this has been the limitation for this project. In order to see the effect of HIS as ground plane of the monopole antenna, two types of HIS is designed. These two HISs will be compared with result obtained from same antenna with $\mathrm{PEC}$ as ground plane.

\section{APPROACH AND METHODOLOGY}

Two types of HIS are designed and built in order to see the differences of HIS with different sizes of the protrusion. See Table I for parameters used to build the HISs.

TABLE I. PARAMETERS OF HIS DESIGN

\begin{tabular}{|c|c|c|}
\hline Parameter & First Design & Second Design \\
\hline Dielectric Constant, $\varepsilon$ & $39.84-\mathrm{pFm}^{-1}$ & $39.84-\mathrm{pFm}^{-1}$ \\
\hline Length, L & $300-\mathrm{mm}$ & $300-\mathrm{mm}$ \\
\hline Height, $\mathrm{H}$ & $300-\mathrm{mm}$ & $300-\mathrm{mm}$ \\
\hline Width, $\mathrm{d}$ & $1.5 \mathrm{~mm}$ & $1.5 \mathrm{~mm}$ \\
\hline Hole's Diameter & $2 \mathrm{~mm}$ & $0.5 \mathrm{~mm}$ \\
\hline Size of each protrusion & $20 \times 20 \mathrm{~mm}$ & $40 \times 40 \mathrm{~mm}$ \\
\hline Gap, g & $0.5 \mathrm{~mm}$ & $0.5 \mathrm{~mm}$ \\
\hline
\end{tabular}

The HIS is designed using Advance Design System and Computer Simulation Technology (CST). The fabrication of HIS is done by a chemical process in the fabrication lab when the layout of designed HISs is converted into Gerber file.

After fabrication is done, each of the protrusion is drilled manually according to the diameter of hole as stated in Table I. Each of the holes is then being soldered in order to make connectivity between top plate with bottom plate.

The HIS is then being attached with $21-\mathrm{cm}$ of monopole antenna that is made up by a rigid copper wire. The antenna and the surface are attached together by a connector which will be used to connect the surface with Network Analyzer for analysis process. See Figure 3. Monopole antenna is selected to be the antenna for HIS because this type of antenna requires a ground plane to be operated besides it is simple to build and design. The performance of the antenna is based on the type of ground plate that is uses. Since this project is to analyze the performance of His as ground plane, it is better to use this type of antenna for HIS analysis.

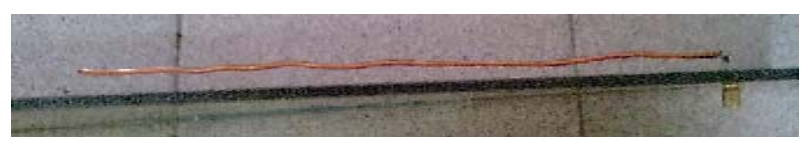

Figure 3. Monopole antenna that is attached to HIS using a connector.

In order to prove the performance of HIS that is believed to enhance the bandwidth, a flat ground plane or perfectly electrically conducting (PEC) plane is also being analyzed with a Network Analyzer after a 21-cm of monopole antenna is being attached to it.

Note that the connector that is used to attach the copper wire as a monopole antenna and to connect the ground plane with Network Analyzer, is put at length 3 inches away from the edge of the ground plane (HIS and PEC). See Figure 4 and Figure 5. The calculation is proved by equation (3) to (4).

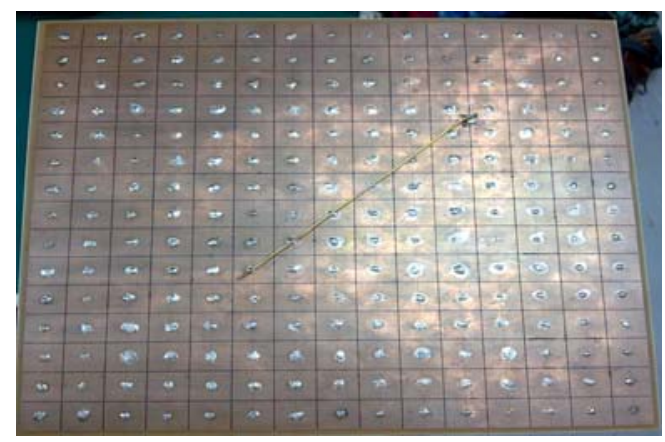

Figure 4. HIS with monopole antenna that is attached to it. 


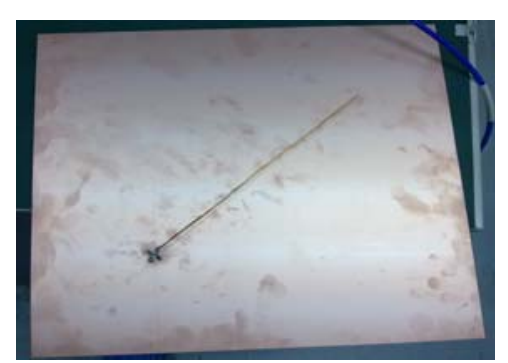

Figure 5. PEC with monopole antenna that is attached to it.

Wavelength, $\lambda=$ Speed of light, $\mathrm{c} /$ Operating frequency, $f$ $=\left(3 \times 10^{8}-\mathrm{m} / \mathrm{s}\right) /(1-\mathrm{GHz})$

$=0.3-\mathrm{m}$

For $\lambda / 4$, the distance will be $=0.3-\mathrm{m} / 4=0.075-\mathrm{m}$

$$
\approx 3 \text {-inch }
$$

The length of copper wire is decided to be as $21-\mathrm{cm}$ because of equation (5). At first, in this project, the antenna in assumed to be operated at $1-\mathrm{GHz}$ due to the maximum frequency of a Network Analyzer, that is why the monopole antenna is came out with length of $21-\mathrm{cm}$.

$$
l=\frac{142.65}{f}
$$

Note that the frequency, $f$, in equation (3) is in Mega Hertz. Therefore, for 1-GHz of frequency, the value of length, $\mathrm{l}$, will be $142.65 / 1000 \mathrm{MHz}$ which yields about $14-\mathrm{cm}$. But in order to come out with a precise conclusion on the analysis that will be done, the length of the rod is extended to 21-cm.

The monopole antenna is put diagonally on the HISs and PEC because at that condition, the rod or the monopole antenna can have a higher contact with the ground plane (HIS and PEC).

Edge of the monopole antenna at the connector side contains maximum current. This current will flow from that edge to the other edge and its value is gradually decreased. At the other edge, the current become minimal. Therefore, it is important to have a better length of monopole antenna. This is because, if the monopole antenna is too long, the current flow is poorer.

For analysis process, the HIS and PEC that are attached with monopole antenna will be measured with Network Anaylzer. The measurement includes S11 only since there is only one port available (one connector). The measurements involve Smith Chart and return loss versus frequency graph. From there, we can measure the bandwidth, frequency, return loss, and impedance matching of the antenna.

After the measurement is done on $21-\mathrm{cm}$ of monopole antenna with HIS as its ground plane, the monopole antenna is being cut to $20-\mathrm{cm}$. And the same measurements are done on it. The antenna is cut every $1-\mathrm{cm}$ and the measurements are recorded. The cutting process is stopped at length of 2$\mathrm{cm}$ since at this point, there is no measurement satisfaction obtained.

The steps of cut and measure are repeated on HIS with different size of patch and on PEC. The analysis data is recorded and shown partially in Table II.

The bandwidth is measured by subtracting the higher frequency with the lower frequency. These two frequencies are selected from return loss graph. From the graph, at any wider $\mathrm{V}$-shapes of line that is also fell below $-10 \mathrm{~dB}$ of return loss will be selected as the best bandwidth. The bandwidth is the difference between to frequencies at $-10 \mathrm{~dB}$ of return loss. See Figure 6 for example of bandwidth calculation.

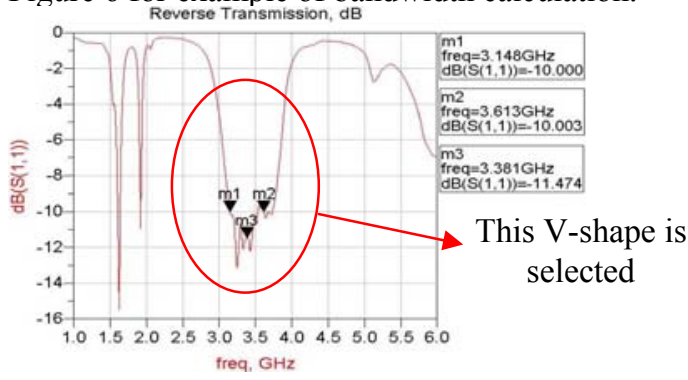

Figure 6. Example of return loss graph for bandwidth calculation.

Values of frequency at $\mathrm{m} 1$ and $\mathrm{m} 2$ will be subtracted and the bandwidth is the yield of the subtracted value. The $m 3$ is the selected frequency for operating frequency of the antenna. The return loss must be below $-10 \mathrm{~dB}$ because of equation (6).

$$
\text { Return loss (dB), RL }=-10 \log _{10} \frac{P_{r}}{P_{t}}
$$

For reflected power, $\mathrm{P}_{\mathrm{r}}$, to be higher that transmitted power, $\mathrm{P}_{t}$, return loss must be higher (if negative sign is not considered) or lower (if negative sign is considered) than -10 $\mathrm{dB}$.

\section{A. Analysis}

The current that is flowed in the monopole antenna is called as real current, $i_{R}$. This current will be reflected back by the ground plane which is the HIS and PEC. Note that the ground plane of a monopole antenna is also a reflector plane that acts a reflector to reflect the current flowed in the monopole antenna. The ground plane induces reflected current or image current, $\mathfrak{i}_{\mathrm{I}}$, of monopole antenna.

For HIS as ground plane, the image current will be in phase and same magnitude as real current. Therefore, the total current will be twice of the real current. This is due to the structure built on top of the plate. Besides, HIS exhibits the same behavior as perfectly magnetically conducting (PMC) plane.

For PEC plane, the image current has same magnitude as real current but it is out of phase with real current. Therefore, the image current will cancel the real current and there will be less or even none current flow for the antenna. 
Result of measurements for HIS with $20 \times 20 \mathrm{~mm}$ size of patch, $40 \times 40 \mathrm{~mm}$ size of patch, and PEC as ground plane are compared in Table IV.

TABLE II. COMPARISON DATA OF HISS AND PEC

\begin{tabular}{|c|c|c|c|c|}
\hline $\begin{array}{c}\text { Types of } \\
\text { Ground } \\
\text { Plate }\end{array}$ & $\begin{array}{c}\text { Length } \\
(\mathbf{c m})\end{array}$ & $\begin{array}{c}\text { Frequency } \\
\text { (GHz) }\end{array}$ & $\begin{array}{c}\text { Return } \\
\text { Loss } \\
\text { (dB) }\end{array}$ & $\begin{array}{c}\text { Bandwidth } \\
\text { (GHz) }\end{array}$ \\
\hline \multirow{2}{*}{$\begin{array}{c}\text { HIS of } \\
\text { 20x20 mm } \\
\text { patch }\end{array}$} & 21 & 5.538 & -17.4 & 0.071 \\
\cline { 2 - 5 } & 13 & 5.469 & -33.547 & 0.076 \\
\hline \multirow{2}{*}{$\begin{array}{c}\text { HIS of } \\
\text { 40x40 mm } \\
\text { patch }\end{array}$} & 21 & 1.605 & -18.889 & 0.1 \\
\cline { 2 - 5 } & 13 & 4.788 & -15.646 & 0.233 \\
\hline \multirow{2}{*}{ Flat } & 21 & 4.525 & -23.805 & 0.309 \\
\cline { 2 - 5 } & 13 & 5.262 & -3.176 & - \\
\cline { 2 - 5 } & 5 & 4.466 & -3.148 & - \\
\hline
\end{tabular}

Those three types of ground plane are analyzed at frequency from $0.1-\mathrm{GHz}$ to $6-\mathrm{GHz}$. This range of frequency is selected because of below equations that give the mathematical (in theory) value of frequency of HIS. Note that $\mathrm{D}$ is sum of length of patch with the size of gap, $\mathrm{g}$.

$$
\text { Capacitance, } C=\frac{\left(\varepsilon+\varepsilon_{o}\right)(D)}{\pi} \ln \left(\frac{2 D}{\pi g}\right)
$$

Inductance, $L=\mu d$

Frequency, $f=\frac{1}{2 \pi \sqrt{L C}}$

From Table V, it shows that HIS with patch size of 40x40 mm has the higher bandwidth with a better return loss. The analysis graphs are shown in Figure 7.

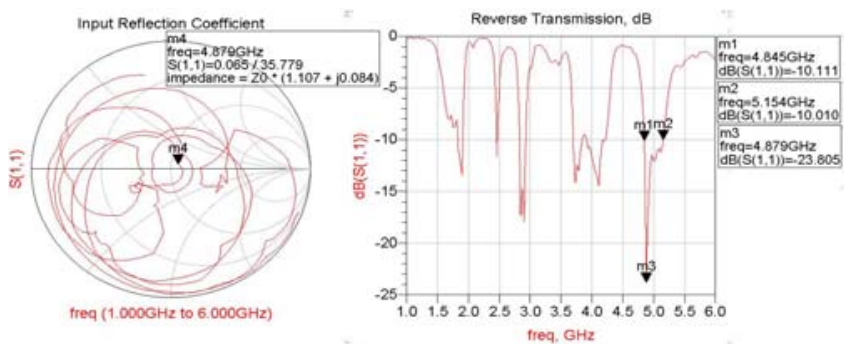

Figure 7. Smith chart and return loss graph of second design of HIS with length of monopole is $13-\mathrm{cm}$.

Through the values of return loss of each type of ground plane, it is proved that the HIS can eliminate the surface waves that reduce the output power since the obtained return loss is lower than $-10 \mathrm{~dB}$. It is due to the gap that is introduced on the plate of HIS. The gap reacts as a filter to block the flow of current. Current that flows on the top of HIS plate cannot be reversed back since there is a path for it to flow which is through the vias to the bottom plate. In normal flat ground plane or PEC, the current has no choice but reversing back to the initial path since the flow of current does not stop. The reverse current also produces the back lobe of power line which also introduces a power leakage.

Surface waves are the power leakage experienced by the plate. It reduces the output power when most the power is radiated away from the plate. With PEC that has a smooth and long surface plat, the current can go any paths it likes. This will cause the power to be radiated away from the plate and cause the power leakage. From here is where the efficiency becomes poorer.

From the tables also we can see that the bandwidth of the HIS is higher than the bandwidth of PEC plane. Note that the bandwidth is the differences between two frequencies at $10 \mathrm{~dB}$ of return loss. Since normal flat plane has no return loss at $-10 \mathrm{~dB}$, the bandwidth is not being calculated and it is assumed to be theoretically minimal.

Figure 8 shows the pattern of graph that is taken from the analysis of PEC with monopole antenna at length of $20-\mathrm{cm}$.

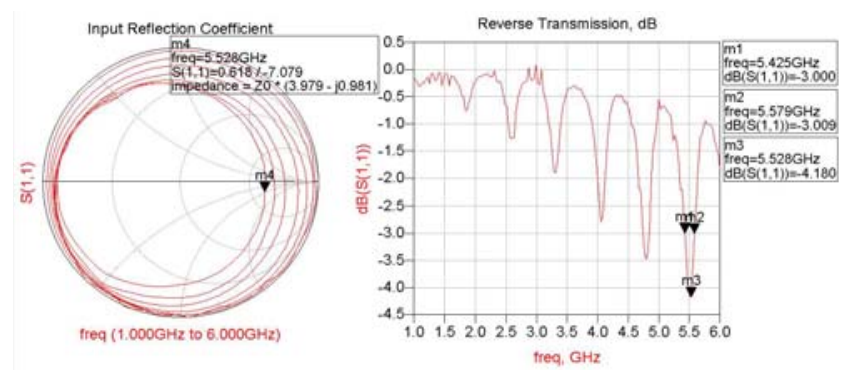

Figure 8. PEC and 20-cm monopole antenna analysis.

From Figure 7, the Smith chart shows that there is impedance mismatching occurred on the antenna. To make it matching, one passive element (capacitor in parallel) is added to the antenna and the results is shown in Figure 9. The capacitor is added through simulation only.

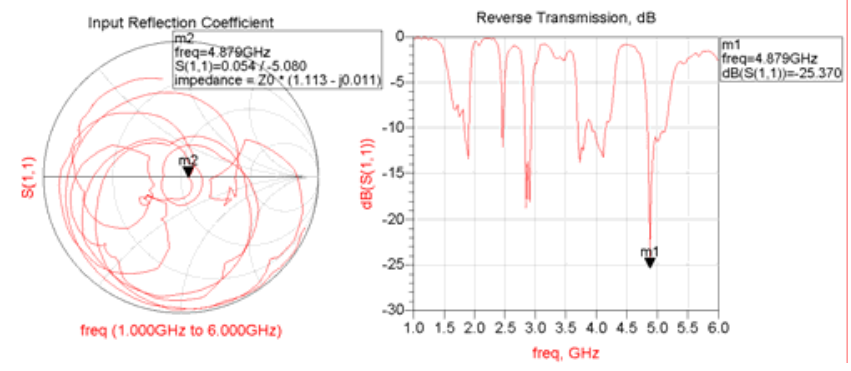

Figure 9. Analysis graphs of HIS (40x40 mm patch) with additional capacitor. 


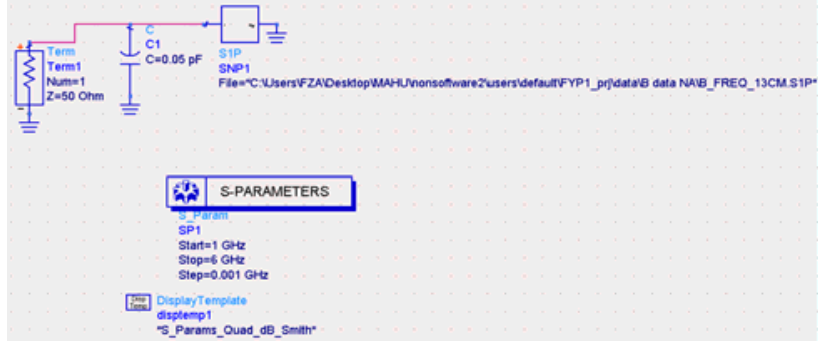

Figure 10. Schematic diagram to obtain simulation graph for additional capacitor.

\section{CONCLUSION}

It is proved that the high impedance surface which is implemented as ground plane can enhance the bandwidth of the antenna as well as other weaknesses such as the return loss and operating frequency. This is proved by comparing the bandwidth and return loss of three different plates.

The flat ground plane did not even achieve the $-10 \mathrm{~dB}$ of return loss. It means that the flat ground plane has a higher value of return loss (if negative sign is considered) or in the other hand, minimum return loss (if negative sign is not considered) which is not good for antenna's performance. Therefore, the value of bandwidth obtained from flat ground plane cannot be compared with the high impedance surface since the return loss did not even touch the $-10 \mathrm{~dB}$. Its bandwidth can be calculated based on $-3 \mathrm{~dB}$ of return loss.

By increasing the size of the patch on HIS, the frequency of the antenna will be decreased. This is because of the value of sheet capacitance that is increased due to the extent of the size. However, the bandwidth will be different base on the length of monopole antenna. This only can be proved by Network Analyzer which can show the performance of the antenna.

The structure of the HIS can reduce the power leakage that is in form of surface waves. The reduction is done by the gap that acts as filter that blocks the flow of current from reversing back to its initial path.

\section{ACKNOWLEDGMENT}

First and foremost, I would like to thank to all of lecturers and supervisors for the valuable and unstoppable guidance and advices. They inspired me greatly to work on this project even though this project needs an extremely commitment from a regular student like me. The willingness of him to motivate me has contributed tremendously to my project which gives a very high credit hour compared to other subjects undertaken.

Besides, I would like to thank to my family, friends, UTP's and UiTM's staffs, and other people who indirectly or directly involved in my project for assisting and providing me with a good support and contributions. It is a great thankful of having all of them who always help me without any hesitations and insincerities. Without helps from them, I might face with many difficulties while doing and completing my project. May God bless of them.

\section{REFERENCES}

[1] David M. Pozar. 20003, Microwave Engineering, United States, John Wiley \& Sons.

[2] Constantine A. Balanis. 1997, Antenna Theory: Analysis and Design, United States, John Wiley \& Sons.

[3] Lee, Kun Sam. 1986, Microstrip Line Leaky Wave Antenna, Ph.D Thesis, University Microfilms International, New York.

[4] Z. I. Dafalla, W. T. Y. Kuan, A. M. Abdel Rahman, and S. C. Shukadar. 2004, Design of a Rectangular Microstrip Patch Antenna at 1 GHz. Report Number 145-149, Multimedia University, Malaysia.

[5] Dan Sievenpiper, Lijun Zhang, Ramulo F. Jimenez Broas, Nicholas G. Alexopolous, Eli Yablonovitch, Nov. 1999, "High Impedance Electromagnetic Surfaces with a Forbidden Frequency Band", IEEE Transactions on Microwave Theory and Technique, Vol.47, No.11.

[6] Dan Qu and Lotfallah Shafai, 2005, "The Performance of Microstrip Patch Antennas over High Impedance EBG Substrates within and outside its Bandgap”, IEEE International Symposium on Microwave, Antenna, Propagation and EMC Technologies for Wireless Communications Proceedings.

[7] Daniel F. Sievenpiper, Joseph L. Pikulsi, James H. Schaffner, and Tsu Yuan Hsu, May 2003, Method of Making a High Impedance Surface, United State Pattern, US 7,197,800 B2.

[8] Gregory J. Dunn, Robert T. Croswell, George H. Kumpf, John A. Svigelj, Jun 2007, High Impedance Electromagnetic Surface and Method. Patent Application Publication, US 2007/0139294 A1. United States.

[9] Telesphor Kamgaing, Omar M. Ramahi, Aug. 2005, "Design and Modeling of High-Impedance Surface for Switching Noise Suppression in Power Planes", IEEE Transactions on Electromagnetic Compatibility, Vol.47, No.3.

[10] Steven R. Best and Drayton L. Hanna, Dec. 2008, "Design of a Broadband Dipole in Close Proximity to an EBG Ground Plane", IEEE Antennas and Propagation Magazine, Vol. 50, No. 6.

[11] Antenna, Lecture Handouts, Course of Communication System Design, Department of Electrical and Computer Engineering, University of New Hampshire, Durham.

[12] Brian Drozd and William T. Joines, May 2004, Comparison of Coaxial Dipole Antennas for Applications in the Near-Field and FarField Regions, Microwave Journal, Duke University, Durham.

[13] Leila Yousefi, Baharak Mohajer-Iravani, and Omar M. Ramahi, "Enhanced Bandwidth Artificial Magnetic Ground Plane for LowProfile Antennas", IEE Antennas and Wireless Propagation Letters, Vol. 6, 2007.

[14] Nader Engheta \& Richard W. Ziolkowaski, "Mathematerials Physics \& Engineering Exploration", IEE Press, 2006, New York.

[15] Fan Yang and Yahya Rahmat-Samii (2009). Electromagnetic Band Gap Structures in Antenna Engineering. New York: Cambridge University Press

[16] Sze, K. Y. (1998). Substrate Thickness in a Microstrip Reflectarry. The 1998 Graduate Student Conference, GRADCON (pp. 4 - 7). Canada: University of Manitoba.

[17] Thomas F Krauss. (2011, January 4). In Wikipedia, The Free Encyclopedia. Retrieved 08:14, March 5, 2012, from http://en.wikipedia.org/w/index.php?title=Thomas_F_Krauss\&oldid= 405880655

[18] E. Yablonovitch, "Inhibited Spontaneous Emission in Solid-State Physics and Electronics", Physical Review Letters, vol. 58, 2059-63, 1987.

[19] Ke Xiao, L.-W. L.-L.-J. (2011). A Novel Monopole-Alike SurfaceWave Antennas Designed Based on Periodic Structures. Antenna Technology (iWAT), 2011 International Workshop (pp. 3 - 7). Hong Kong: Institute of Electrical and Electronics Engineers (IEEE).

[20] Pandya, R. B. (2010, Jun 14). High Impedance Electromagnetic Surfaces and Their Applications. Retrieved December 2011, from docstoc: http://www.docstoc.com/docs/43556937/Presentation-OnHigh-Imp. 